\title{
MANAGEMENT OF URINARY TRACT INFECTION IN ACUTE PARAPLEGIC PATIENTS WITH SPECIAL REFERENCE TO THE PAPER STRIP SCREENING TEST FOR INFECTION
}

\author{
By J. R. Silver, M.B., B.S., M.R.C.P. \\ National Spinal Injuries Centre, Stoke Mandeville Hospital
}

THE present study reports the management of urinary tract infection in Ioo consecutive acute spinal injury patients treated on an acute male ward at the National Spinal Injuries Centre, over a period of 2 years, advocates the early detection of infection by use of a screening test.

Immediately after an acute spinal injury the majority of the patients require drainage of the bladder. Intermittent catheterisation was introduced at the National Spinal Injuries Centre, Stoke Mandeville Hospital, soon after its foundation in 1944 by Sir Ludwig Guttmann. It was described by him in 1947 and I953. Detailed results obtained on 476 patients have been presented by Guttmann and Frankel (I966) and later by Walsh (I968) on 92 patients. It is accepted throughout the world that this is the best initial form of treatment. Pearman (I97I), Ott and Rossier (I97I) and Miller (I97I) have extended the technique and confirmed the excellent results.

Pearman (I97I) achieved his excellent results by the closest bacteriological surveillance and cultured every specimen of urine taken, since by the time urine turbidity appeared or pyrexia had occurred, urinary infection had probably been present for at least 48 hours. It was apparent that the former method of culturing urine once a week or when infection was suspected, was not satisfactory and it was necessary to do a screening test for urinary infection on every specimen of urine that was taken from the patients on intermittent catheterisation.

\section{METHOD}

Culture and identification was carried out by the methods of Cowan and Steel (1965). The method of intermittent catheterisation described by Guttmann and Frankel (I966) has been followed with some modifications.

Following the work of Stykler et al. (I97I), it was considered that the anterior urethra was the source of ascending infection. An attempt was made to sterilize the urethra by filling it with $\frac{1}{2}$ per cent. Hibitane in glycerine from an individual sterile bottle for each patient prior to catheterisation. This solution is rather sticky and if a pre-lubricated catheter is used, the combination sometimes blocks the catheter, and so a dry catheter is now used.

Screening Test for Urinary Infection. The method described by Leigh and Williams (I964) was employed. A piece of sterile filter paper (dipstrips, Mast laboratories) is dipped into the freshly taken urine using aseptic precautions. Time is given for the excess fluid to be absorbed and then an impression of the angulated end of the paper $\frac{1}{2}^{\prime \prime} \times \frac{1_{1}^{\prime \prime}}{1}$ in size is made on the surface of a well-dried MacConkey agar plate. Urine from six patients can be inoculated onto one appropriately labelled plate which is then incubated (figs. I and 2). 


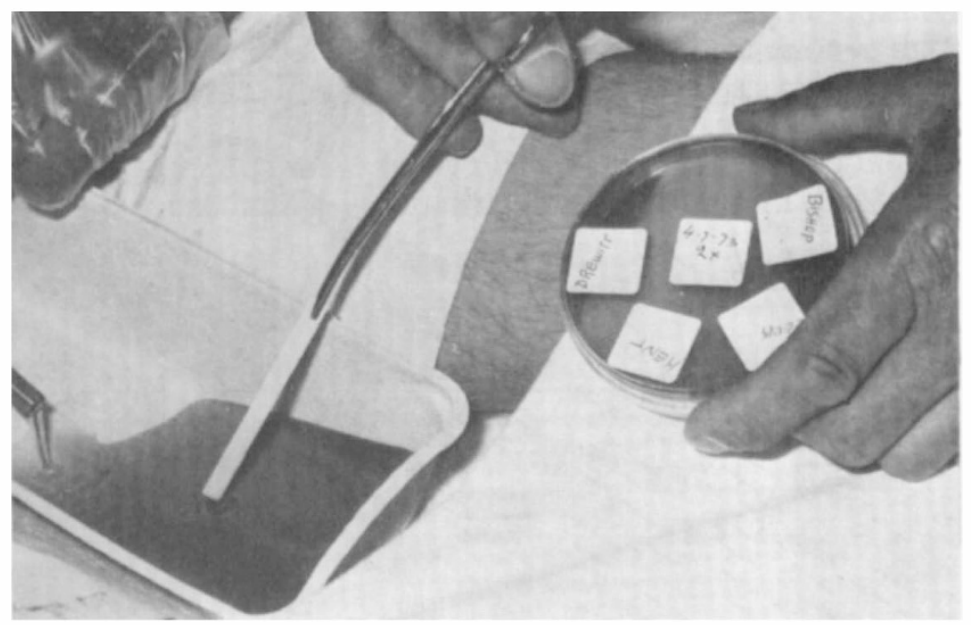

FIG. I

Standard filter paper dipped in urine after catheterisation. The plate has previously been labelled on the reverse side.

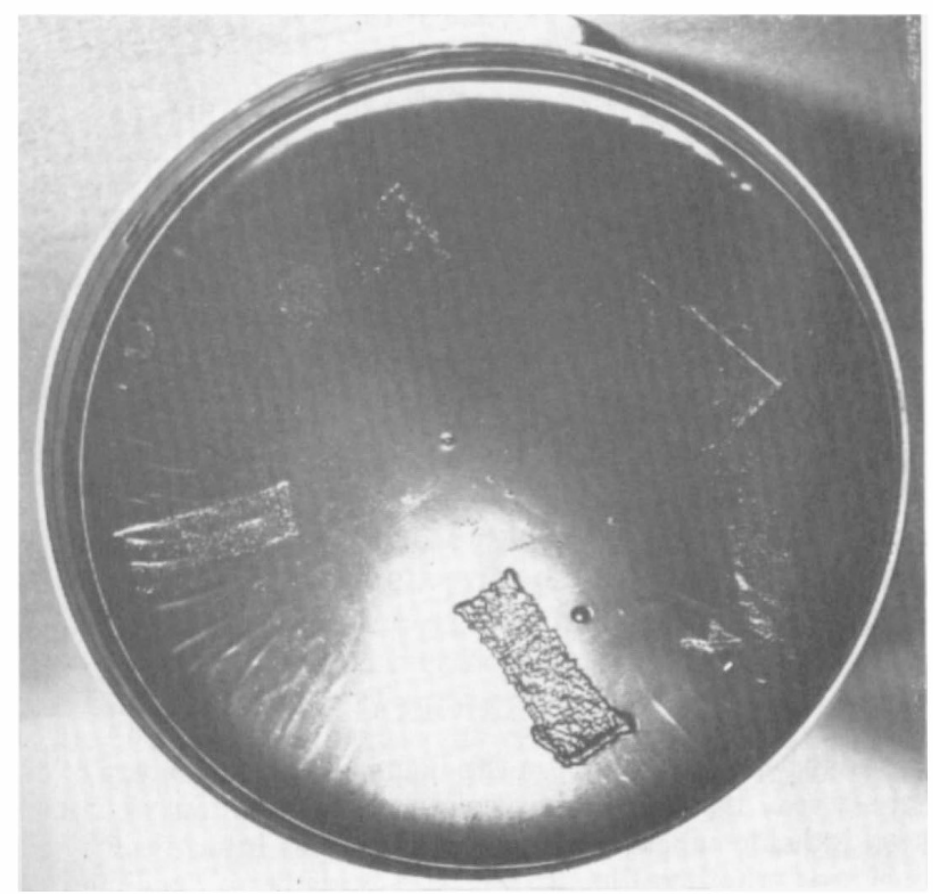

FIG. 2

Heavy confluent growth on one area. The other inoculations can barely be seen, unless the plate is angled to catch the light. 
Infection was judged to be present the next day if two consecutive inoculations show a growth of 20 or more colonies, representing $10^{5}$ or more organisms per $\mathrm{ml}$. A specimen of urine was also sent for microscopy and culture on every patient in the ward once a week (fig. 3).

This technique was initially carried out on every specimen of urine taken on patients undergoing intermittent catheterisation. Unfortunately, at night as one

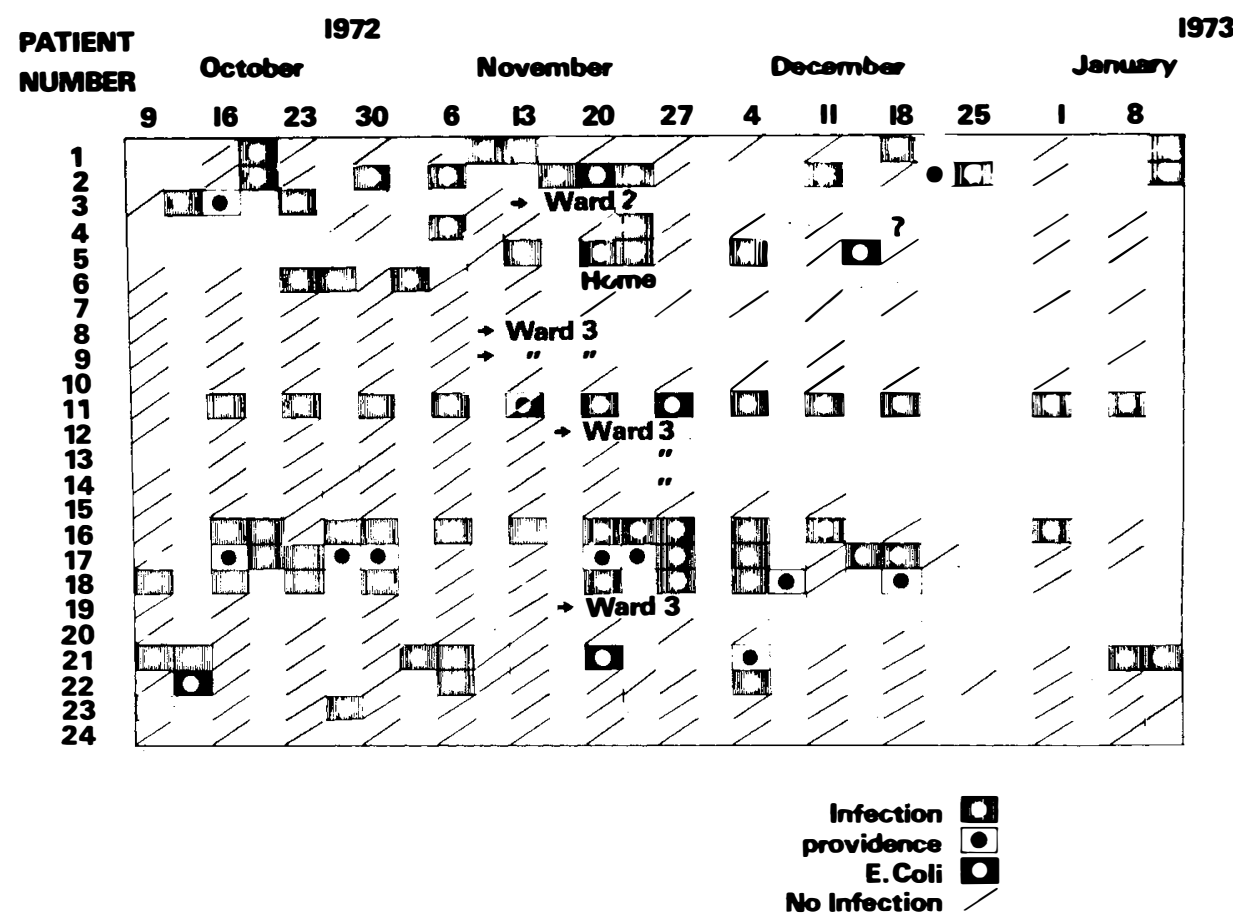

FIG. 3

Diagram of a section of the chart showing the infection on a 23-bedded ward. With the use of colours every infection can be separately plotted and at a glance the incidence, distribution and speed of eradication of infection noted.

doctor does the catheters on several wards with different catheter orderlies, confusion arose too frequently in the correct plating of specimens. Accordingly, the night plating was stopped.

\section{BACTERIOLOGY}

In August 1970 a chart was kept in the Bacteriology laboratory of every urinary infection in the ward, using different colours to plot different bacterial species and symbols to indicate whether the patient was on intermittent or continuous catheter drainage or whether the urine was a midstream specimen. There was room to record two specimens a week for each patient (fig. 3). The chart enabled one to see the number of infections in the ward and the predominant causative organism each week. The MacConkey plate screening test was introduced in 
February 1972. A separate simple chart was kept recording the result of each test, with three symbols indicating 'No growth' 'New infection' or 'Continuing infection' (fig. 4). Only the details of new infections were recorded on the 'Urine report cards'. These cards are kept in the Pathology Office and photocopies are sent to the ward.

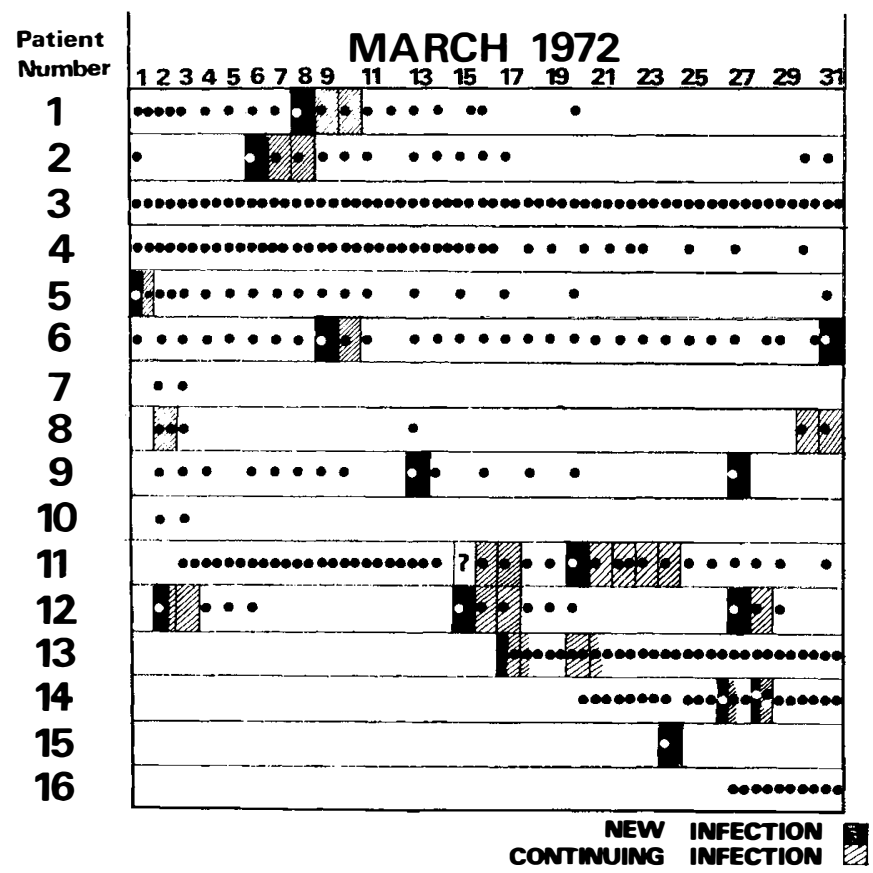

FIG. 4

Diagram of the screening test chart. Each . represents a single catheterisation. Two catheterisations are plotted per day, although three are carried out.

Note patient 3 remains free of infection for many days. Also the speed of eradication of infection.

Routine Management. Before the intermittent catheters were passed in the morning the screening test plates were assessed, and taking into account residual urine, one of the following regimes was instituted.

Continuation of intermittent catheterisation, indwelling catheter, bladder washouts with local instillation of antibiotics or antiseptics, or systemic antibiotic treatment without waiting for sensitivity test results.

If the MacConkey plate showed an infection, the organism was identified and sensivitity tests done. The next day, when sensitivities were known, the report was sent to the ward and there might be a discussion between the bacteriologist and the clinician as to the correct antibiotic for an individual patient. Once a week on a Thursday, there would be a joint round in the Bacteriology Department in which all the urinary infections would be assessed in conjunction with the amount of residual urine and the treatment, and the policy with regard to treatment and 
investigation for the forthcoming week would be discussed. In general the policy for treatment was decided on this round.

\section{PATIENTS}

A hundred male patients, aged between II and 79, were studied between April I97I and March 1973. The ward in which they were studied had 23 beds. For clinical details see Table I.

\section{TABLE I}

Clinical details of patients (I5 April I97I to 17 March 1973)

\begin{tabular}{|lc|c|c|}
\hline Level of lesion & Cervical & Thoracic & Lumbar \\
\hline Complete & 3 I & 24 & I \\
Incomplete & 20 & I 4 & IO \\
\hdashline Totals & 5 I & 38 & I I \\
\hline
\end{tabular}

Two deaths occurred during this period. Seventy-eight of the patients were under the age of 40 . Fifty-nine of the patients were admitted within 7 days of injury.

Results (Table II). Fifty-nine patients had sterile urine on admission, 4I patients had infected urine on admission. At the end of the period studied, I7 March 1973, 86 patients had sterile urine, I2 patients had infected urine. These results are encouraging in that more were sterile than at the time of admission.

Overall Incidence of Infection on the Ward from August 1970. During the nine months before the investigation urinary infection reached a peak of I6 out of 23 patients. Regular weekly meetings instituted in May I97I resulted in a rapid fall in infection and further improvement followed the introduction of the urethral antiseptic, increased dosage of G.500 and the introduction in February I972 of the screening test. Deterioration occurred with the appearance of new doctors on the ward unfamiliar with all aspects of the management of the technique of intermittent catheterisation (fig. 5).

Speed of Acquisition of Infection. Evaluation is more accurate if the daily screening test is employed. Of 46 patients 19 had become infected within 6 days whereas others could continue on intermittent catheterisation for as long as 44 days without infection, but half the patients were infected by Io days. Infection may have already been acquired when catheterisation was carried out elsewhere and antibiotic cover instituted (fig. 6).

Total Number of Infections. The total number of new infections in the patients on intermittent catheterisation during the period of study was $2 \mathrm{II}$.

There was no correlation with the month of the year when these new infections were acquired, the numbers of new infections ranging from 5 to 28 per month 
TABle II

Details of Ioo patients' urine on admission and discharge with infection and management 
at random, but there was a very marked correlation in the days of the week when the infections were first detected. The numbers being as follows:

52 on Monday; 35 on Tuesday; 28 on Wednesday; 36 on Thursday; 26 on Friday; 7 on Saturday; 37 on Sunday.

The higher numbers seen on Sunday and Monday are a reflection of poor management over the week-ends when there is a shortage of doctors carrying out the intermittent catheterisations. This was borne out by one particular week-end

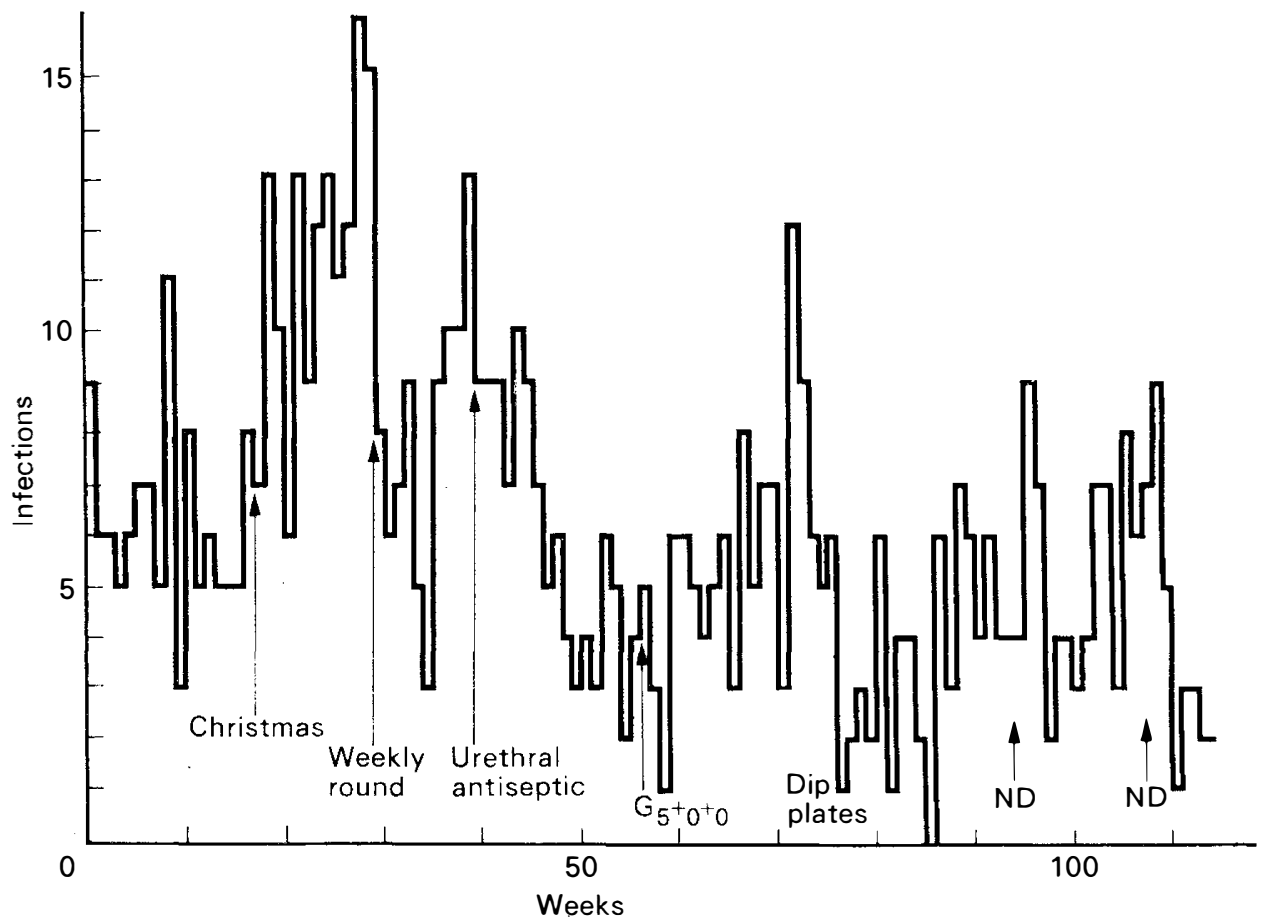

FIG. 5

$\mathrm{G}++=$ increased dosage of $\mathrm{G} .500$.

$\mathrm{ND}=$ new ward doctor.

During the 9 months before the collaborative effort began the chart showed a high incidence of urinary infection which reached a peak during the early months of $197 \mathrm{I}$. The highest level was I6 out of 23 patients infected, just before there was a change in medical staffing. With the introduction of the regular weekly round in May I97I, there was a rapid fall in the infection, reflecting more prompt treatment of the infections as they were detected, but there was a gradual rise again with a dramatic fall following the introduction of the urethral antiseptic in the technique in June 1970. Following this there was a change with regard to the policy in the use of G.500. The dosage was increased to the full amount previously recommended, $4 \times 4$ per day and all patients not on antibiotics were treated, and the overall results at the time showed a mean number of infections considerably less than previously. But again the infections crept up to a maximum of 12 on one occasion, with a dramatic fall once again in February 1971/72 with the introduction of the screening tests. In 3 weeks subsequently there was only one infection on the ward and in I week, no infections, whereas prior to the collaborative effort three infections was the least, which was only found once, and the norm was five. There were several smaller peaks of infection on the ward when new doctors came to the Centre. 
when seven out of nine patients on intermittent catheterisation acquired fresh infections.

Speed of Elimination of Infection. With the introduction of the screening test all the infections were eliminated within 3 days of onset and thus prolonged periods free of infection could be achieved. This was made possible because new infections were discovered within 24 hours of onset.

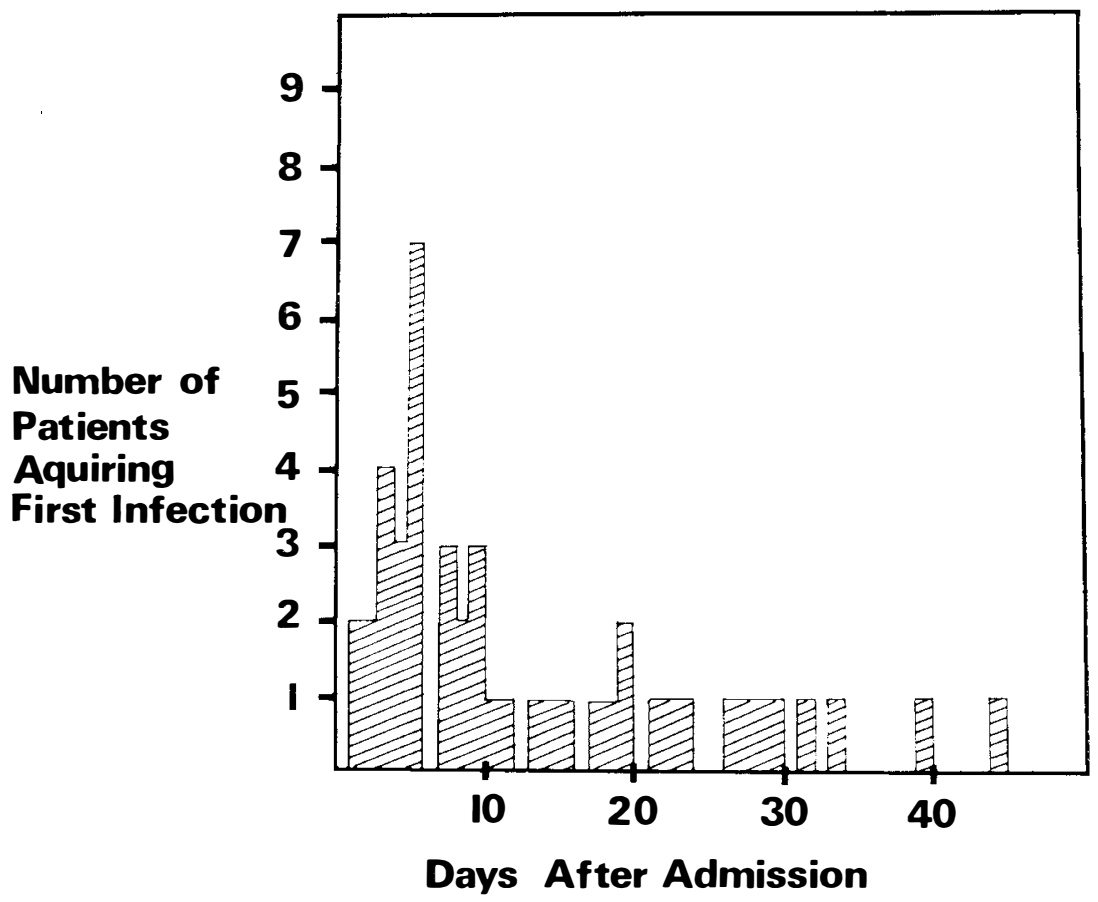

Fig. 6

Diagram showing the speed with which 46 patients admitted with sterile urine acquired their first infection.

Type of Organism. Of the 59 patients admitted with sterile urine the first infections detected in 56 were as follows:

I9 Klebsiella aerogenes; 6 Strep. faecalis; 6 Pseudomonas pyocyanea; I4 Proteus; I E. freundi; 4 Providence; 3 E. coli; 3 Aerobacter cloacae.

Many of these were hospital acquired organisms. An interesting point was the change in the distribution of the organisms over a period of time. In August 1970, there were two Providence infections resistant to all current antibiotics, which rose to a maximum of seven in February I97I. By July I97I, with the introduction of the closer surveillance, the resistant Province virtually disappeared.

Complications. Hydronephrosis. Eight of the patients had hydronephrosis, only two being admitted early with sterile urine. The other six patients were 
admitted later between $\mathrm{I} O$ and $5 \mathrm{I}$ days after injury, with heavily infected urine and indwelling catheters. One patient had heavy haematuria. The bladders had not been managed adequately, catheters had been changed infrequently and were encrusted with stones.

Reflux. Five of the patients had reflux, two of these having hydronephrosis already described above. Three patients who had reflux but no hydronephrosis were all admitted late after injury at 6 weeks, 2 months and 3 months. These patients had an identical picture of unsatisfactory bladder management, i.e. infected urine and rare changes of catheter.

Septicaemia. Of this group of I I patients, three had septicaemia, one before admission and two shortly after discharge. Two others of this group of I I patients have died at home. In both cases the cause of death was given as heart failure. There is close correlation with the figures from the larger series of Guttmann and Frankel who found $7 \cdot 4$ per cent. had hydronephrosis, compared with 8 per cent. and 4.4 per cent. reflux, compared with 5 per cent. in this group.

\section{DISCUSSION}

The results are encouraging compared with those achieved by Guttmann and Frankel who admitted 80.7 per cent. sterile and discharged 64.3 per cent. sterile out of 409 males. Walsh admitted 92 per cent. of his patients with sterile urine and discharged 82.6 per cent. sterile out of a series of 92 males. In the present series of IOO patients only 59 per cent. had sterile urine on admission and 86 per cent. had sterile urine on discharge or on I7 March 1973 although 37 have not yet been discharged. The series is smaller than that of Guttmann and Frankel and has not been followed up as long. However, these results are not as good as Pearman's, who over a 2-year period admitted 36 patients and discharged 33 with sterile urine. Rossier dealt with 34 men and discharged $3 \mathrm{I}$ with sterile urine. It is impressive that Pearman's group of patients had as an average only one infection every two patients throughout the study, whereas only is patients in our series were free of infection throughout the study and many had more than one infection. In Pearman and Rossier's studies, they were dealing with a smaller number of patients and the catheters were passed by orderlies. It is clear that the major factor in the successful management is the care with which the bladder is dealt with and the urine monitored, since with each improvement in techniquethe weekly round, the urethral antiseptic, the use of screening test-there was a fall in the incidence of infection. The converse is indicted by the high incidence of infection acquired over the week-end when one doctor has to carry out all the catheterisation on the unit.

On admission patients are not prophylacticly treated with antibiotics. It is believed, contrary to the experience of Pearman, that the use of systemic antibiotics only depresses the natural defence mechanisms and does not prevent urinary infection, the only difference being that the organism is resistant to the antibiotics used. Many patients receive antibiotics for the treatment of their intercurrent chest complications and when the urine becomes infected in individual cases it is resistant to that antibiotic.

Management of infections by intermittent catheterisation, antibiotics and local washouts, is potentially dangerous in that an organism can multiply within the bladder and enter into the blood stream in the period between catheterisations. 
This is particularly so in the case of flare-ups when there may well be transient reflux; in this case fluid intake is increased, washouts are not given, and in severe cases an indwelling catheter is inserted. The findings in this series would tend to substantiate the view that hydronephrosis and reflux are not caused by neurogenic factors but infection, since all but two of the patients with these complications were admitted with severe urinary infections, and the first cystogram and I.V.P. showed hydronephrosis and reflux. Presumably damage due to infection had occurred at the ureteric orifices, resulting in the reflux or obstruction giving rise to hydronephrosis. If a patient is admitted more than 3 weeks after injury with an indwelling catheter continuous drainage is not discontinued immediately, the catheter is changed, a urine culture, an I.V.P., a cystogram, cystometrogram and if necessary a cystoscopy is performed before starting intermittent catheterisation while the inevitable urinary infection is treated by the appropriate antibiotic. The danger of hydronephrosis and reflux is brought out by the observation that three of these patients developed septicaemia. The deaths of two other patients with hydronephrosis at home from heart failure may well have been contributed to by infection.

The absolute numbers of patients with infected urine on a ward will be affected by the admission policy as well as by the treatment of the infection. In August 1970 two wards were closed through lack of nursing staff and it was not possible to admit the patients as early as formerly. This is reflected in the fact that $4 \mathrm{I}$ of these patients were admitted with infected urine and only 59 were admitted within 7 days of injury. The presence of a large number of patients with infected urine on a ward tends to potentiate infection on the ward since it is impossible for the ward doctor to manage too many catheters by the technique of intermittent catheterisation and washouts and he is thus more likely to contaminate other patients. When there is a heavy infection with a resistant organism, such as Providence, large dosage of antibiotics to which the organism is only partially sensitive are not given. In such cases the residual urine is eliminated by putting a catheter in the bladder, all antibiotics and antiseptics are discontinued and time is allowed to elapse for the bladder to be colonised by a less resistant organism. When this has taken place, if there is no systemic involvement and the catheter is to be left in, the organism is usually stable and the patient can remain off antibiotics with the catheter in. Alternatively, the catheter can be removed under the cover of a suitable antibiotic and the programme of intermittent catheterisation recommended. When a new infection is detected on the MacConkey plate screening test and if the patient is ill, we usually start treatment with the most likely affective antibiotic, while awaiting the sensitivity test results the next day in order to eliminate the organism as quickly as possible. The results here show that the infection has been eliminated within 2 days. Surprisingly as a result of this policy the infections acquired by the patients on the ward have been sensitive to Sulphonamide, Furadantin and Negram. Having eliminated the infection the patient commences a urinary antiseptic, i.e. G.500. Once there is a large residual urine and the bladder does not empty completely following an adequate period of bladder training ( 12 weeks in bed and say another 3-4 weeks to enable gravity to help the bladder, aided by the use of Carbachol) infection once established cannot be eliminated. O'Grady and Cattell have shown that the residual urine which is infected serves as a sump to reinfect fresh urine secreted by the kidneys. Surgery (Table III) is, therefore, carried out as appropriate on the 


\section{TABLE III}

Details of 18 patients who had surgery upon their bladders

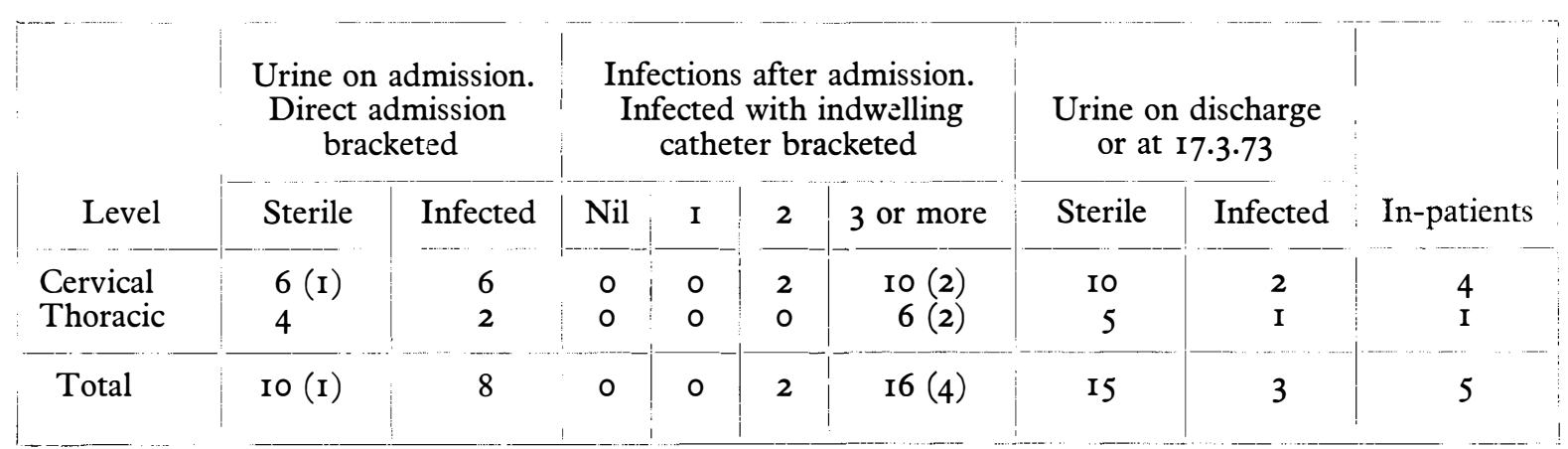

Notes: I cervical patient with an infected urine later died.

I cervical patient later required an indwelling catheter and developed stones.

I cervical patient had his operation at his first check-up readmission. 
bladder neck or external sphincter to improve drainage and the results in this group are excellent, there being I 5 patients out of I 8 with sterile urine as a result of surgery. In addition to using the dip plate technique on acute injuries it has been used in the later stages for bladder training following a transurethral resection or when a patient develops a fresh or recurrent infection late after injury and can be used on a small number of acute medical admissions such as transverse myelitis and enables treatment to be monitored much more quickly.

Surveillance of infection has been facilitated by the use of the screening test and can now be regarded as an integral part of the management which can be carried out at any time of day or night, both to diagnose an infection and to monitor treatment. Culture and microscopy of many specimens of urine is laborious and time consuming. The use of a screening test has been labour saving in the laboratory and beneficial to the patient. There is obviously much to be done to improve management as we have a higher incidence of infection than that reported by Pearman; he recorded one infection every two patients, compared with this series where only I 5 out of Ioo patients remained sterile and the lesson is that it is not one technique that is the answer but it is the attention to every detail in the management of the bladder that can achieve better results.

The meticulous attention to trivialities make perfection and perfection is no triviality.

\section{SUMMARY}

The application of a paper strip screening test for urinary infection in the management of paraplegic patient is described. In acute injuries cultures are carried out daily on specimens of urine. A hundred consecutive patients admitted between I5 April I97I and I7 March 1973 are reviewed. There were 5I cervical, 38 thoracic and II lumbar lesions. Fifty-nine were admitted within 7 days of injury. On admission 59 patients had sterile urine, $4 \mathrm{I}$ had infected urine. Two patients died during the study. At the end of the period or on discharge 86 patients had sterile urine. Half the patients with sterile urine acquired their first infection by Io days. It was acquired on a Sunday or Monday in the majority of cases and eliminated in 2 days. The advantages of this technique in the speed of elimination of the infection and the maintenance of the urine in a sterile condition are discussed and comparisons made with other series.

Acknowledgements. I would like to thank Dr. A. E. Dike for carrying out the bacteriology and for her help and assistance throughout the preparation of this paper. I would also like to thank Dr. C. L. Greenbury, Director of the Pathology Laboratory and Dr. J. J. Walsh, Director of the National Spinal Injuries Centre, for their encouragement and help.

\section{REFERENCES}

Cowan, S. T. \& Steel, K. J. (1965). Identification of Medical Bacteria. Cambridge University Press.

Guttmann, L. (1947). Proc. Royl. Soc. Med. 40, 219.

Guttmann, L. (I953). History of Second World War. Volume Surgery, ed. Cope, p. 422. H.M.S.O.

Guttmann, L. and Frankel, H. (1966). Paraplegia, 4, 63.

Leigh, D. A. \& Williams, J. D. (I964). F. Clin. Path. I 7, 498. 
Miller, J. M. (I97I). Proceedings of the I8th Veterans Administration Spinal Cord Injury Conference, p. I48.

O'GRADY, F. \& CATTELl, W. R. (1966). Brit. F. Urol., p. I56.

OTT, R. \& Rossier, A. B. (I97I). Proceedings of the I8th Veterans Administration Spinal Cord Injury Conference, p. 139.

Pearman, J. W. (I97I). Proceedings of the I8th Veterans Administration Spinal Cord Injury Conference, p. II 5 .

StykleR, D. J., Wilmot, C. B. \& O'FlynN, J. D. (1970). The mode of development of urinary infection in intermittently catheterised male paraplegics. Int. F. Paraplegia, 8, 243.

Walsh, J. J. (I968). Paraplegia, 6, 74.

\section{Discussion}

Sir Ludwig Guttmann (G.B.). I congratulate Dr. Debarge and his colleagues on their interesting research on plasma catecholamines in tetraplegics, with their refined techniques. Although the stimuli applied by the authors to tetraplegics were somewhat different from our previous experiments on bladder distension, they confirm that the rise of blood pressure is not the result of a primary increase of catecholamines, in particular noradrenaline, but is the result of nervous reflex activity as has been found previously. There is now more proof that the reflex response on the sympathetic system below the level of cord transection above $\mathrm{T}_{5}$, and in particular in tetraplegics, due to bladder percussion or electrical stimulation of the muscles is the reflex response which is responsible for the hypertension of both the systolic and diastolic blood pressure. As we found previously in these cervical lesions, the reflex vasoconstriction affects the vascular bed of the greater part of the body and is primarily responsible for the particularly high values of the hypertension in these high lesions. Although the recently developed technique of Christensen in assessing plasma catecholamines may be more accurate than that described in our previous publications on this subject, the principle of the reflex response on the nervous system was well established in our previous experiments. From the figures shown, it is quite clear that the effect of bladder hyperactivity on blood pressure was more marked than that of the stimulation of the skeletal muscles. Of special interest to me was the effect of noradrenaline infusion on blood pressure which showed a much more marked response in tetraplegics than in the normal controls.

With regard to Silver and Dike's paper, I was interested to hear that there was a difference in the results of intermittent catheterisation in the early stages when this was done during the week-end, as compared with other days of the week. I feel that in the acute stages, there should be no difference in the meticulous technique whether it is done during the week-end or on any other day of the week. I feel very strongly that it is the duty of the senior physician or surgeon in charge of the case to ensure that the technique is carried out with the same skill and meticulous care.

DR. WA TSON (G.B.). Just a comment on Dr. Silver's paper. The question is about that organism called Providence. Is it B. coli or a variation of Proteus? Perhaps Dr. Silver can tell me? Intermittent catheterisation is being practised here and I agree that it is the best method, but here at Stoke Mandeville, the patients are having antiseptics put down the urethra and antiseptics swallowed by mouth and quite frankly the bacteriological results are rather difficult to evaluate. It may be that the constant use of all these antiseptic agents perhaps has led to the evolution of this more resistant organism that you have in your Centre.

Professor A. Rossier (U.S.A.). I would like to make a point on what was just said. Most of the patients at Geneva were off antibiotics except for pulmonary complications, where they received penicillin which is known not to be basically a urinary drug, and our results on intermittent catheterisation were exactly the same, of the order of 86 to 88 percent.

DR. P. Dollfus (France). In the Accident Unit in Colmar, where intermittent 
catheterisation is practised, it is performed by nurses. This is done up to five or sometimes six times in 24 hours. We do not use any antibiotics at all except very rarely, and the urine remains sterile for an average of 4 to 6 weeks before we start using an antibiotic. We consider that antibiotics are dangerous and we do not use them unless we are absolutely sure of what we are using them against.

Dr. J. Silver (G.B.). With regard to Dr. Watson's point, unfortunately everybody refers to it as Dr. Silver's paper, but it is Silver and Dike's paper. Dr. Dike is a bacteriologist who has done most of the work of recording and correlating. She would have given the correct answer to Dr. Watson's question about Providence, but had to leave earlier. I agree with Dr. Watson that resistant organisms are the result of the antibiotic therapy. The point I was trying to make was that we do not routinely treat patients with antibiotics and I think there is a certain defence mechanism in the body and that if you give powerful poisons you depress this mechanism. With regard to sterilising the urethra with antiseptics, it may be our fault that we are not leaving the antiseptic in for long enough. Perhaps we should leave it in for Io to 15 minutes before we pass the catheter. I don't believe that this has any effect on the flora within the bladder, you just sterilise the infected area of the urethra.

Dr. Watson. I still don't know what Providence is as you haven't told me. You have been talking about Providence here for years and I still do not know what you mean by it.

Dr. H. Frankel (G.B.). I can give an explanation of what Province is. It is a para-colon bacillus which in most laboratories is only reported as such. It is a Gramnegative bacillus somewhere near to the Proteus group and it is an organism which occurs almost entirely in a hospital environment where there are many antibiotics in use.

Chairman (Dr. H. TAlbot (U.S.A.)). If I may take advantage of the prerogative of the chair, I think we should try to break down the insidious and dangerous habit of indiscriminate and prolonged applications of antibiotics immediately you see a pus cell or an organism. This has probably killed as many people in the long run as antibiotics have saved over the decades since I 94I. I think that the point made by Dr. Dollfus with his extraordinary good results without antibiotics is something we should all take to heart.

Mr. Griffiths (Australia). I think that Providence must be one that has immigrated from the U.S.A. I have known Providence for many years and have never, like Dr. Watson, asked what organism it was. I have always thought it was a $B$. coli or a strain of $B$. coli.

DR. MEINECKe (Germany). I would like to make some comments on the paper by Mr. Burr and Dr. Walsh. For some years we have been doing X-rays of the kidneys and bladder as soon as possible to exclude stone formations in the urinary tract which have developed before the onset of paraplegia. If it is possible we also do at the same time an I.V.P.

Recently I received a letter from a firm in Germany which propagated a tidal drainage machine recommended by a urologist, who used it for bladder trouble in bladder cancer. He also recommends this machine for spinal cord injuries. Moreover, he recommends to train a spastic bladder with low capacity to become larger. I have written to the firm saying that this gentleman obviously has no knowledge about the problem of tidal drainage in these cases, and we should warn our colleagues to read this paper with great criticism.

ChaIRMan. A great deal of unfortunate material has been published by urologists who have not been thoroughly acquainted with the problem of spinal cord injuries. This is a very real problem and I don't know the solution. There is always a tendency for people who want to use a gadget which has a magic effect and think you just hook it up and the problem is solved. 